\title{
High-Throughput Computation of Thermal Conductivity of High-Temperature Solid Phases: The Case of Oxide and Fluoride Perovskites
}

\author{
Ambroise van Roekeghem, ${ }^{1, *}$ Jesús Carrete, ${ }^{1}$ Corey Oses, ${ }^{2}$ Stefano Curtarolo, ${ }^{2,3}$ and Natalio Mingo ${ }^{1}$ \\ ${ }^{1}$ CEA, LITEN, 17 Rue des Martyrs, 38054 Grenoble, France \\ ${ }^{2}$ Center for Materials Genomics, Duke University, Durham, North Carolina 27708, USA \\ ${ }^{3}$ Materials Science, Electrical Engineering, Physics and Chemistry, \\ Duke University, Durham, North Carolina 27708, USA \\ (Received 13 June 2016; published 21 December 2016)
}

\begin{abstract}
Using finite-temperature phonon calculations and machine-learning methods, we assess the mechanical stability of about 400 semiconducting oxides and fluorides with cubic perovskite structures at 0,300 , and $1000 \mathrm{~K}$. We find 92 mechanically stable compounds at high temperatures_including 36 not mentioned in the literature so far-for which we calculate the thermal conductivity. We show that the thermal conductivity is generally smaller in fluorides than in oxides, largely due to a lower ionic charge, and describe simple structural descriptors that are correlated with its magnitude. Furthermore, we show that the thermal conductivities of most cubic perovskites decrease more slowly than the usual $T^{-1}$ behavior. Within this set, we also screen for materials exhibiting negative thermal expansion. Finally, we describe a strategy to accelerate the discovery of mechanically stable compounds at high temperatures.
\end{abstract}

DOI: 10.1103/PhysRevX.6.041061

\section{INTRODUCTION}

High-throughput ab initio screening of materials is a new and rapidly growing discipline [1]. Amongst the basic properties of materials, thermal conductivity is a particularly relevant one. Thermal management is a crucial factor to a vast range of technologies, including power electronics, CMOS interconnects, thermoelectric energy conversion, phase change memories, turbine thermal coatings, and many others [2]. Thus, rapid determination of thermal conductivities for large pools of compounds is a desirable goal in itself, which may enable the identification of suitable compounds for targeted applications. A few recent works have investigated thermal conductivity in a highthroughput fashion [3,4]. A drawback of these studies is that they were restricted to using $0 \mathrm{~K}$ phonon dispersions. This is often reasonable when the room-temperature phase is mechanically stable at $0 \mathrm{~K}$. However, it poses a problem for materials whose room- or high-temperature phase is not the $0 \mathrm{~K}$ structure: when dealing with structures exhibiting displacive distortions, including temperature effects in the phonon spectrum is a crucial necessity.

Such a phenomenon is prevalent for perovskites. Indeed, the perovskite structure can exhibit several distortions from

\footnotetext{
*ambroise.van-roekeghem@polytechnique.edu
}

Published by the American Physical Society under the terms of the Creative Commons Attribution 4.0 International license. Further distribution of this work must maintain attribution to the author(s) and the published article's title, journal citation, and DOI.
Subject Areas: Chemical Physics,

Computational Physics,

Materials Science

the ideal cubic lattice, which are often responsible for rich phase diagrams. When the structure is not stable at low temperatures, a simple computation of the phonon spectrum using forces obtained from density functional theory (DFT) and the finite displacement method yields imaginary eigenvalues. This prevents us from assessing the mechanical stability of these compounds at high temperatures or calculating their thermal conductivities. Moreover, taking into account finite-temperature effects in phonon calculations is currently a very demanding task, especially for a high-throughput investigation.

In this study, we are interested in the high-temperature properties of perovskites, notably for thermoelectric applications. For this reason, we focus on perovskites with the highest symmetry cubic structure, which are most likely to exist at high temperatures [5-9]. With the help of the highthroughput AFLOW framework [10,11], we explore a chemical space containing more than 7000 compounds (see Supplemental Material [12]), and first screen for paramagnetic semiconductors using standard density functional theory calculations [13]. This yields 391 compounds, for which we include the effects of anharmonicity in our $a b$ initio calculations of mechanical and thermal properties.

\section{FINITE-TEMPERATURE CALCULATIONS OF MECHANICAL STABILITY AND THERMAL PROPERTIES}

Recently, several methods have been developed to deal with anharmonic effects at finite temperatures in solids [14-19]. In this study, we use the method presented in 
Ref. [19] to compute the temperature-dependent interatomic force constants, which uses a regression analysis of forces from DFT coupled with a harmonic model of the quantum canonical ensemble. This is done in an iterative way to achieve self-consistency of the phonon spectrum. The workflow is summarized in Fig. 1, and is referred to as SCFCS - standing for self-consistent force constants. As a trade-off between accuracy and throughput, we choose a $3 \times 3 \times 3$ supercell and a cutoff of $5 \AA$ for the third-order force constants. Special attention is paid to the computation of the thermal displacement matrix [19], due to the imaginary frequencies that can appear during the convergence process, as well as to the size of the supercell that normally prevents us from sampling the usual soft modes at the corners of the Brillouin zone (see Supplemental Material [12]). This allows us to assess the stability at $1000 \mathrm{~K}$ of the 391 hypothetical compounds mentioned in Sec. I. Among this set, we identify 92 mechanically stable compounds, for which we also check the stability at $300 \mathrm{~K}$. The phonon spectra of the stable compounds are provided in the Supplemental Material [12]. Furthermore, we compute the thermal conductivity using the finite-temperature force constants and the full solution of the Boltzmann transport equation as implemented in the ShengBTE code [20].

We list the stable compounds and their thermal conductivities in Fig. 2. Remarkably, this list contains 37

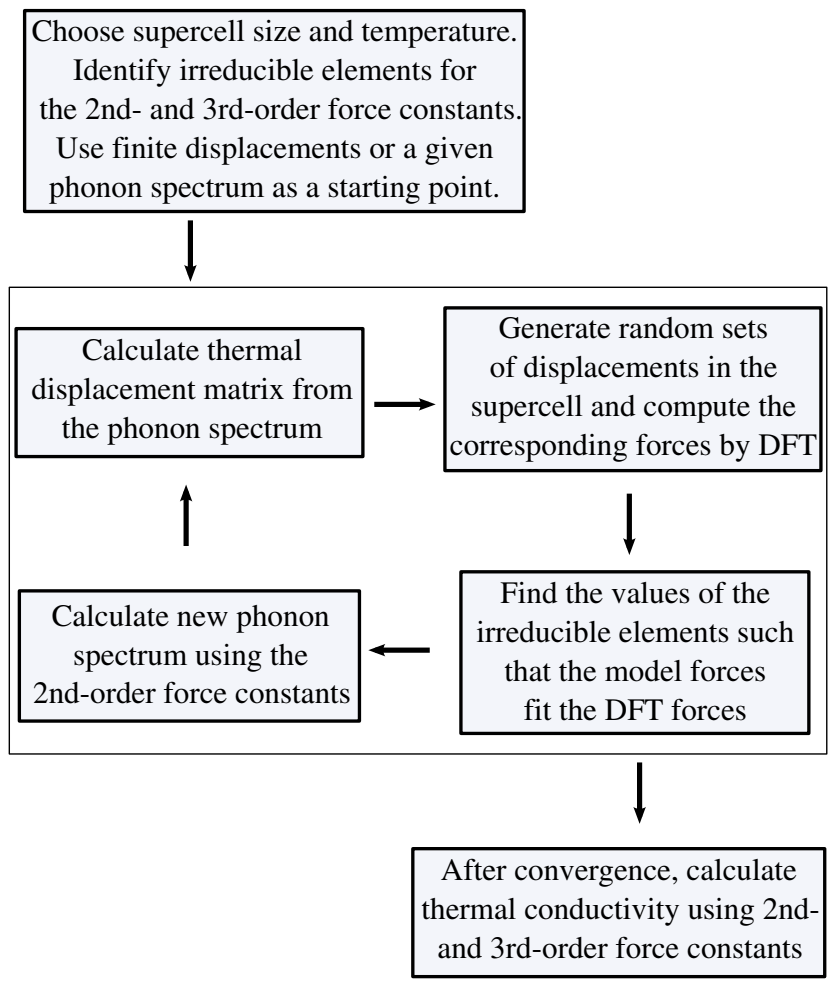

FIG. 1. Workflow of the method used to calculate the phonon spectrum and thermal conductivity including finite-temperature anharmonic effects. perovskites that have been reported experimentally in the ideal cubic structure (see references in Fig. 2), which lends support to our screening method. On the other hand, we also find that 11 compounds are reported only in a nonperovskite form. This is not necessarily indicative of mechanical instability, but instead suggests thermodynamical stability may be an issue for these compounds, at least near this temperature and pressure. Thirty-six compounds remain unreported experimentally in the literature to our knowledge. Thus, by screening only for mechanical stability at high temperatures, we reduce the number of potential new perovskites by a factor of 10 . Furthermore, we find that 50 of them are mechanically stable in the cubic form close to room temperature.

Of the full list of perovskites, only a few measurements of thermal conductivity are available in the literature. They are displayed in parentheses in Fig. 2 along with their calculated values. Our method tends to slightly underestimate the value of the thermal conductivity, due to the compromises we make to limit the computational cost of the study (see Supplemental Material [12]). This discrepancy could also be partially related to the electronic thermal conductivity, which is not subtracted in the measurements. Still, we expect the order of magnitude of the thermal conductivity and the relative classification of different materials to be consistent. More importantly, this large data set allows us to analyze the global trends driving thermal conductivity. These trends are discussed in Sec. IV.

We also investigate the (potentially) negative thermal expansion of these compounds. Indeed, the sign of the coefficient of thermal expansion $\alpha_{V}$ is the same as the sign of the weighted Grüneisen parameter $\gamma$, following $\alpha_{V}=\left(\gamma c_{V} \rho\right) / K_{T}$, where $K_{T}$ is the isothermal bulk modulus, $c_{V}$ is the isochoric heat capacity, and $\rho$ is the density $[84,85]$. The weighted Grüneisen parameter is obtained by summing the contributions of the mode-dependent Grüneisen parameters: $\gamma=\sum \gamma_{i} c_{V i} / \sum c_{V i}$. Finally, the mode-dependent parameters are related to the volume variation of the mode frequency $\omega_{i}$ via $\gamma_{i}=$ $-\left(V / \omega_{i}\right)\left(\partial \omega_{i} / \partial V\right)$. In our case, we calculate those parameters directly using the second- and third-order force constants at a given temperature $[16,86,87]$ :

$$
\gamma_{m}=-\frac{1}{6 \omega_{m}^{2}} \sum_{i j k \alpha \beta \gamma} \frac{\epsilon_{m i \alpha}^{*} \epsilon_{m j \beta}}{\sqrt{M_{i} M_{j}}} r_{k}^{\gamma} \Psi_{i j k}^{\alpha \beta \gamma} e^{i \mathbf{q} \cdot \mathbf{r}_{j}}
$$

This approach has been very successful in predicting the thermal expansion behavior in the empty perovskite $\mathrm{ScF}_{3}$ [19], which switches from negative to positive around $1100 \mathrm{~K}$ [88]. In our list of filled perovskites, we find only two candidates with negative thermal expansion around room temperature, $\mathrm{TlOsF}_{3}$ and $\mathrm{BeYF}_{3}$, and none at $1000 \mathrm{~K}$. This shows that filling the perovskite structure is probably detrimental to the negative thermal expansion. 


\begin{tabular}{|c|c|c|c|c|c|c|c|c|c|c|c|c|c|c|}
\hline & $\kappa_{1000}$ & & $\kappa_{300}$ & & References & & $\kappa_{1000}$ & $\kappa_{300}$ & & References & & $\kappa_{1000}$ & $\kappa_{300}$ & References \\
\hline $\mathrm{CaSiO}_{3}$ & 4.89 & & & & {$[21]$} & $\mathrm{CdYF}_{3}$ & 1.29 & 3.51 & & & $\mathrm{TlOsF}_{3}$ & 0.62 & 0.95 & \\
\hline $\mathrm{RbTaO}_{3}$ & 3.61 & & & & [22] & $\mathrm{RbCaF}_{3}$ & 1.15 & 2.46 & $(3.2)$ & {$[23-25]$} & $\mathrm{InZnF}_{3}$ & 0.61 & 1.86 & \\
\hline $\mathrm{NaTaO}_{3}$ & 3.45 & & & & [26] & $\mathrm{HgInF}_{3}$ & 1.15 & 3.85 & & & $\mathrm{CsCdF}_{3}$ & 0.59 & 1.73 & {$[27]$} \\
\hline $\mathrm{CuCF}_{3}$ & 3.32 & & 8.79 & & [28] & $\mathrm{AlFeF}_{3}$ & 1.14 & & & & $\mathrm{AlMgF}_{3}$ & 0.56 & & \\
\hline $\mathrm{SrSiO}_{3}$ & 3.23 & & 10.10 & & [29] & $\mathrm{PbHfO}_{3}$ & 1.12 & & & {$[30]$} & $\mathrm{AuZnF} \mathrm{Zn}_{3}$ & 0.53 & & \\
\hline $\mathrm{NaNbO}_{3}$ & 3.05 & & & $(1.5)$ & [31-33] & $\mathrm{AgMgF}_{3}$ & 1.11 & & & {$[34]$} & $\mathrm{InOsF}_{3}$ & 0.52 & & \\
\hline $\mathrm{BaHfO}_{3}$ & 3.04 & $(4.5)$ & 8.26 & $(10.4)$ & [35] & $\mathrm{ZnScF}_{3}$ & 1.10 & 3.66 & & & $\mathrm{RbSrF}_{3}$ & 0.51 & & {$[36]$} \\
\hline $\mathrm{KNbO}_{3}$ & 2.94 & & & $(10)$ & {$[31,33]$} & $\mathrm{RbFeF}_{3}$ & 1.09 & 4.62 & & {$[37]$} & $\mathrm{CsSrF}_{3}$ & 0.50 & 1.13 & {$[36]$} \\
\hline $\mathrm{TlTaO}_{3}$ & 2.86 & & & & {$[38]$} & $\mathrm{TlMgF}_{3}$ & 1.06 & 3.42 & & {$[39]$} & $\mathrm{BeYF}_{3}$ & 0.48 & 2.34 & \\
\hline $\mathrm{AgTaO}_{3}$ & 2.77 & & & & {$[40,41]$} & $\mathrm{KCaF}_{3}$ & 1.06 & & & {$[42]$} & $\mathrm{BeScF}_{3}$ & 0.48 & 1.59 & \\
\hline $\mathrm{KMgF}_{3}$ & 2.74 & & 8.25 & $(10)$ & {$[25,43]$} & $\mathrm{HgScF}_{3}$ & 1.01 & 5.42 & & & $\mathrm{TlCdF}_{3}$ & 0.44 & & {$[27]$} \\
\hline $\mathrm{GaTaO}_{3}$ & 2.63 & & & & {$[44-46]$} & $\mathrm{CsCaF}_{3}$ & 0.98 & 3.03 & & {$[47]$} & $\mathrm{RbHgF}_{3}$ & 0.43 & & {$[48]$} \\
\hline $\mathrm{BaTiO}_{3}$ & 2.51 & & 4.99 & $(4-5)$ & {$[33,49]$} & $\mathrm{AuMgF}_{3}$ & 0.96 & & & & $\mathrm{PdYF}_{3}$ & 0.43 & 0.99 & \\
\hline $\mathrm{PbTiO}_{3}$ & 2.42 & & & $(5)$ & [33] & $\mathrm{InMgF}_{3}$ & 0.96 & 3.53 & & & $\mathrm{AlZnF}_{3}$ & 0.39 & & \\
\hline $\mathrm{SrTiO}_{3}$ & 2.36 & $(4)$ & 6.44 & $(10.5)$ & {$[51-53]$} & $\mathrm{RbZnF}_{3}$ & 0.91 & 2.64 & & {$[54]$} & $\mathrm{KHgF}_{3}$ & 0.37 & & {$[48]$} \\
\hline $\mathrm{SrHfO}_{3}$ & 2.20 & $(2.7)$ & & $(5.2)$ & {$[53,55]$} & $\mathrm{ZnInF}_{3}$ & 0.88 & 1.89 & & & $\mathrm{RbSnF}_{3}$ & 0.37 & 0.82 & {$[56]$} \\
\hline $\mathrm{BaZrO}_{3}$ & 2.13 & $(2.9)$ & 5.61 & $(5.2)$ & [57] & $\mathrm{BaSiO}_{3}$ & 0.87 & & & {$[58]$} & $\mathrm{ZnBiF}_{3}$ & 0.37 & 1.29 & \\
\hline $\mathrm{XeScF}_{3}$ & 1.87 & & 4.40 & & & $\mathrm{TlCaF}_{3}$ & 0.86 & & & & $\mathrm{CsHgF}_{3}$ & 0.37 & 1.00 & {$[48]$} \\
\hline $\mathrm{HgYF}_{3}$ & 1.84 & & 5.37 & & & $\mathrm{CdScF}_{3}$ & 0.85 & 2.37 & & & $\mathrm{KSnF}_{3}$ & 0.35 & & {$[56]$} \\
\hline $\mathrm{AgNbO}_{3}$ & 1.79 & & & & {$[59,60]$} & $\mathrm{XeBiF}_{3}$ & 0.82 & 2.13 & & & $\mathrm{CdBiF}_{3}$ & 0.33 & 0.98 & \\
\hline $\mathrm{TlNbO}_{3}$ & 1.75 & & & & [38] & $\mathrm{AgZnF}_{3}$ & 0.80 & & & {$[34]$} & $\mathrm{RbPbF}_{3}$ & 0.32 & & [61] \\
\hline $\mathrm{KFeF}_{3}$ & 1.72 & & 6.37 & $(3.0)$ & {$[62,63]$} & $\mathrm{PdScF}_{3}$ & 0.79 & 1.63 & & & $\mathrm{BeAlF}_{3}$ & 0.30 & 1.70 & \\
\hline $\mathrm{SnSiO}_{3}$ & 1.66 & & 4.22 & & {$[64,65]$} & $\mathrm{KCdF}_{3}$ & 0.75 & & & {$[66,67]$} & $\mathrm{KPbF}_{3}$ & 0.30 & & {$[68]$} \\
\hline $\mathrm{PbSiO}_{3}$ & 1.66 & & 3.69 & & {$[69,70]$} & $\mathrm{BaLiF}_{3}$ & 0.73 & 2.21 & b & {$[71,72]$} & $\mathrm{CsBaF}_{3}$ & 0.29 & & \\
\hline $\mathrm{AuNbO}_{3}$ & 1.56 & & & & [73] & $\mathrm{HgBiF}_{3}$ & 0.72 & 2.37 & & & $\mathrm{InCdF}_{3}$ & 0.29 & & \\
\hline $\mathrm{CaSeO}_{3}$ & 1.42 & & & & [74] & $\mathrm{ZnAlF}_{3}$ & 0.72 & 1.92 & & & $\mathrm{BaCuF}_{3}$ & 0.28 & & \\
\hline $\mathrm{NaBeF}_{3}$ & 1.40 & & 2.53 & & {$[75,76]$} & $\mathrm{GaZnF}_{3}$ & 0.69 & & & & $\mathrm{TlSnF}_{3}$ & 0.27 & 0.63 & {$[77]$} \\
\hline $\mathrm{RbMgF}_{3}$ & 1.37 & & 4.54 & & {$[78]$} & $\mathrm{RbCdF}_{3}$ & 0.68 & 1.46 & & {$[27]$} & $\mathrm{TlHgF}_{3}$ & 0.26 & & [79] \\
\hline $\mathrm{GaMgF}_{3}$ & 1.34 & & 2.11 & & & $\mathrm{GaRuF}_{3}$ & 0.67 & & & & $\mathrm{CdSbF}_{3}$ & 0.26 & & \\
\hline $\mathrm{KZnF}_{3}$ & 1.33 & & 4.15 & $(5.5)$ & {$[25,80]$} & $\mathrm{CsZnF}_{3}$ & 0.67 & 1.12 & & [81] & $\mathrm{TlPbF}_{3}$ & 0.22 & & {$[82]$} \\
\hline $\mathrm{ZnYF}_{3}$ & 1.32 & & 3.72 & & & $\mathrm{TlZnF}_{3}$ & 0.64 & 1.96 & & [83] & & & & \\
\hline
\end{tabular}

a $\mathrm{AuMgF}_{3}$ was mentioned theoretically in Ref. [50].

b The thermal diffusivity of $\mathrm{BaLiF}_{3}$ was measured at $300 \mathrm{~K}$ in Ref. [71] as $\alpha=0.037 \mathrm{~cm}^{2} \mathrm{~s}^{-1}$.

FIG. 2. List of cubic perovskites found to be mechanically stable at $1000 \mathrm{~K}$ and their corresponding computed lattice thermal conductivity (in $\mathrm{W} \mathrm{m}^{-1} \mathrm{~K}^{-1}$ ). We also report the computed lattice thermal conductivity at $300 \mathrm{~K}$ (in $\mathrm{W} \mathrm{m}^{-1} \mathrm{~K}^{-1}$ ) when we obtain stability at that temperature. We highlight in blue the compounds that are experimentally reported in the ideal cubic perovskite structure, and in red those that are reported only in nonperovskite structures (references provided in the table). When no reference is provided, no mention of the compound in this stoichiometry has been found in the experimental literature. Experimental measurements of the thermal conductivity are reported in parentheses, and in italics when the structure is not cubic.

We also examine the evolution of the thermal conductivity as a function of temperature for the compounds that are mechanically stable at 300 and $1000 \mathrm{~K}$. There is substantial evidence that the thermal conductivity in cubic perovskites generally decreases more slowly than the model $\kappa \propto T^{-1}$ behavior $[89,90]$ at high temperatures, in contrast to the thermal conductivity of, e.g., Si or Ge, that decreases faster than $\kappa \propto T^{-1}$ [91]. This happens, for instance, in $\mathrm{SrTiO}_{3}[51,52], \mathrm{KZnF}_{3}[25,80], \mathrm{KMgF}_{3}$ [25], $\mathrm{KFeF}_{3}$ [63], $\mathrm{RbCaF}_{3}$ [25], $\mathrm{BaHfO}_{3}$ [35], $\mathrm{BaSnO}_{3}$ [92], and $\mathrm{BaZrO}_{3}$ [57]. We also predicted an anomalous behavior in $\mathrm{ScF}_{3}$ using ab initio calculations, tracing its origin to the important anharmonicity of the soft modes [19]. Figure 3 displays several experimentally measured thermal conductivities from the literature on a logarithmic scale, along with the results of our high-throughput calculations. As discussed above, the absolute values of the calculated thermal conductivities are generally underestimated, but their relative magnitude and the overall temperature dependence are generally consistent. Although the behavior of the thermal conductivity $\kappa(T)$ is in general more complex than a simple power-law behavior, we model the deviation to the $\kappa \propto T^{-1}$ law by using a parameter $\alpha$ that describes approximately the temperature dependence of $\kappa$ between 300 and $1000 \mathrm{~K}$ as $\kappa \propto T^{-\alpha}$. For instance, in Fig. 3, $\mathrm{KMgF}_{3}$ appears to have the fastest decreasing thermal conductivity with $\alpha=0.9$ both from experiment and calculations, while $\mathrm{SrTiO}_{3}$ is closer to $\alpha=0.6$. At present, there are too few experimental measurements of the thermal conductivities in cubic perovskites to state that the $\kappa \propto T^{-\alpha}$ behavior with $\alpha<1$ is the general rule in this family. However, the large number of theoretical predictions provides a way to assess this trend. Of the 50 compounds that we find to be mechanically stable at room 


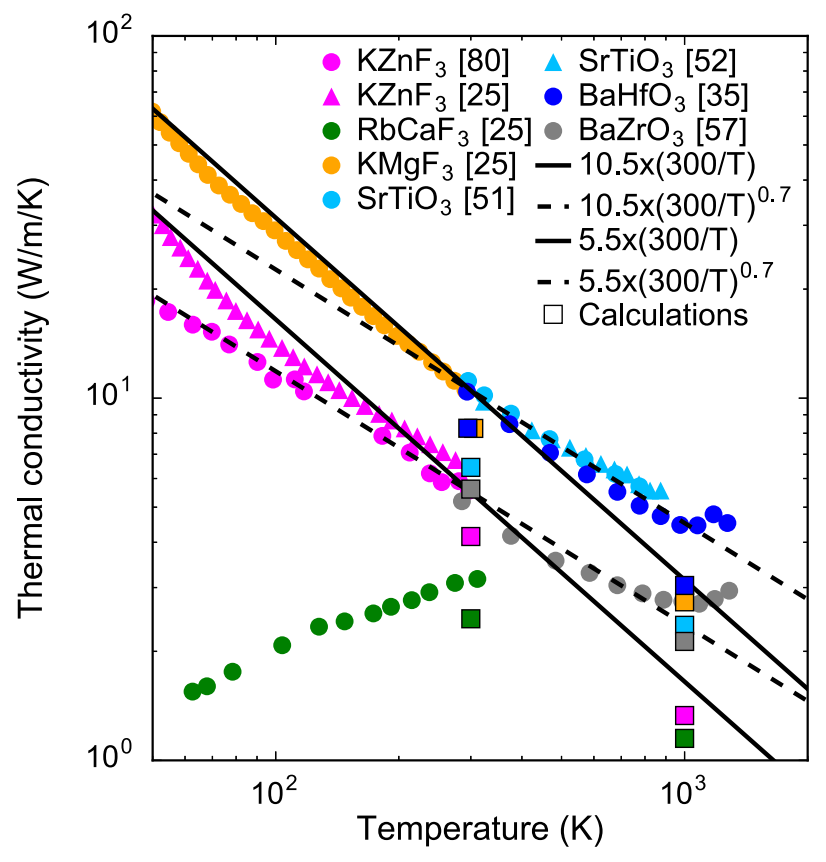

FIG. 3. A comparison between total thermal conductivities from Refs. [25,35,51,52,57,80], high-throughput calculations of the lattice thermal conductivity at 300 and $1000 \mathrm{~K}$, and model behaviors in $\kappa \propto T^{-1}$ and $\kappa \propto T^{-0.7}$.

temperature, we find a mean $\alpha \simeq 0.85$, suggesting that this behavior is likely general and correlated to structural characteristics of the perovskites.

\section{ACCELERATING THE DISCOVERY OF STABLE COMPOUNDS AT HIGH TEMPERATURE}

Through brute-force calculations of the initial list of 391 compounds, we extract 92 that are mechanically stable at $1000 \mathrm{~K}$. However, this type of calculation is computationally expensive. Thus, it is desirable for future highthroughput searches of other material classes to define a strategy for exploring specific parts of the full combinatorial space. In this section, we propose and test such a strategy based on an iterative machine-learning scheme using principal component analysis and regression.

We begin by calculating the second-order force constants $\Phi_{0 \mathrm{~K}}$ of all compounds using the finite-displacement method, which is more than an order of magnitude faster than finite-temperature calculations. This gives us a list of 29 perovskites that are mechanically stable in the cubic phase at $0 \mathrm{~K}$. Since this is the highest symmetry phase, they are likely also mechanically stable at high temperatures [93]. We calculate their self-consistent finite-temperature force constants $\Phi_{1000 \mathrm{~K}}^{\mathrm{SCFC}}$, as described in Sec. II. This initial set allows us to perform principal component analysis (PCA) of the $0 \mathrm{~K}$ force constants so that we obtain a transformation that retains the 10 most important components. In a second step, we use regression analysis to find a relation between the principal components at 0 and at

\begin{tabular}{|c|c|}
$\begin{array}{c}\text { Set of stable compounds } \\
\text { at } 1000 \mathrm{~K} \text { and force constants } \\
\text { at } 0 \mathrm{~K} \text { and } 1000 \mathrm{~K}\end{array}$ & $\rightarrow \quad \begin{array}{c}\text { Train PCA and regression } \\
\text { of force constants } \\
\text { from } 0 \mathrm{~K} \text { to } 1000 \mathrm{~K}\end{array}$ \\
\hline
\end{tabular}

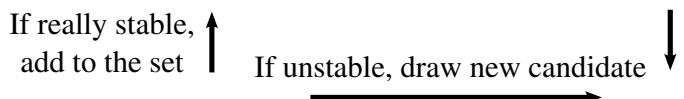

Perform full calculation to verify stability and obtain the force constants at $1000 \mathrm{~K}$
Find new candidate for stability at $1000 \mathrm{~K}$ using the model
FIG. 4. Depiction of strategy for exploring the relevant combinatorial space of compounds that are mechanically stable at high temperature.

$1000 \mathrm{~K}$. This finally gives us a model that extracts the principal components of the force constants at $0 \mathrm{~K}$, interpolates their values at $1000 \mathrm{~K}$, and reconstructs the full force constants matrix at $1000 \mathrm{~K}, \Phi_{1000 \mathrm{~K}}^{\text {model }}$. We say that this model has been "trained" on the particular set of compounds described above. Applying it to the previously calculated $\Phi_{0 \mathrm{~K}}$ for all compounds, we can efficiently span the full combinatorial space to search for new perovskites with a phonon spectrum that is unstable at $0 \mathrm{~K}$ but stable at $1000 \mathrm{~K}$. For materials determined mechanically stable with $\Phi_{1000 \mathrm{~K}}^{\mathrm{model}}$, we calculate $\Phi_{1000 \mathrm{~K}}^{\mathrm{SCFCS}}$. If the mechanical stability is confirmed, we add the new compound to the initial set and subsequently train the model again with the enlarged set. When no new compounds with confirmed mechanical stability at high temperatures are found, we stop the search. This process is summarized in Fig. 4. Following this strategy, we find 79 perovskites that are stable according to the model, 68 of which are confirmed to be stable by the full calculation. This means that we have reduced the total number of finite-temperature calculations by a factor of 5 , and that we have retrieved mechanically stable compounds with a precision of $86 \%$ and a recall of $74 \%$ [95]. It allows us to obtain approximate phonon spectra for unstable compounds, which is not possible with our finitetemperature calculations scheme (see Supplemental Material [12]). It also allows us to find compounds that had not been identified as mechanically stable by the first exhaustive search due to failures in the workflow. Considering the generality of the approach, we expect this method to be applicable to other families of compounds as well. Most importantly, it reduces the computational requirements, particularly if the total combinatorial space is much larger than the space of interest.

\section{SIMPLE DESCRIPTORS OF THE THERMAL CONDUCTIVITY}

We now focus on the analysis of the thermal conductivity data provided in Fig. 2. We note that this set contains about 2 times more fluorides than oxides. This is already the case 
after the first screening in which we keep only the semiconductors, and it can be explained by the strong electronegativity of fluorine, which generally forms ionic solids with the alkali- and alkaline-earth metals easily, as well as with elements from groups 12,13, and 14. This is shown in Fig. 5, in which we display histograms of the columns of elements at sites $A$ and $B$ of the perovskite in our initial list of paramagnetic semiconductors and after screening for mechanical stability.

We can also see that the oxides tend to display a higher thermal conductivity than the fluorides, as shown in the density plot of Fig. 6. This is once again due to the charge of the fluorine ion, which is half that of the oxygen ion. In a model of a purely ionic solid, this would cause the interatomic forces created by electrostatic interactions to be divided by two in fluorides as compared to oxides. This is roughly what we observe in our calculations of the second-order force constants. It translates into smaller phonon frequencies and mean group velocities in fluorides as compared to oxides. Fluorides also have smaller heat capacities, due to their larger lattice parameters (see Supplemental Material [12]). Those two factors mainly drive the important discrepancy of the thermal conductivity between fluorides and oxides. Following the same reasoning, it means that halide perovskites, in general, should have a very low thermal conductivity.

Finally, we analyze the correlations between the thermal conductivity and different simple structural descriptors.
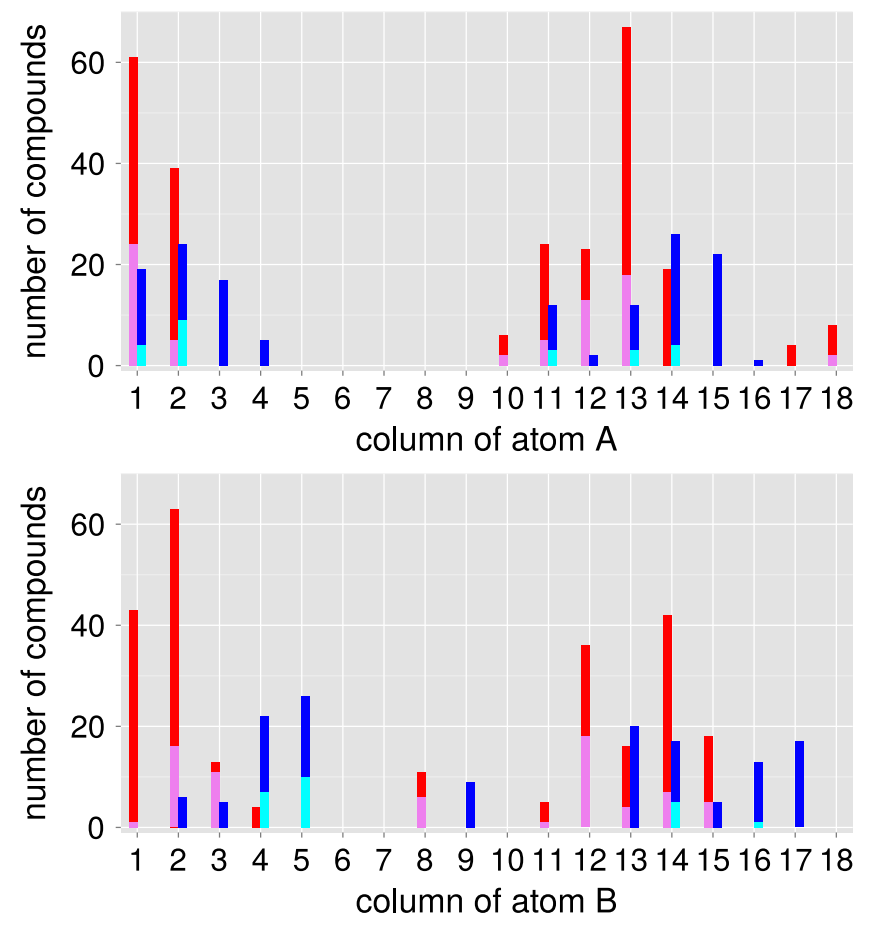

FIG. 5. Column number of the element at site $A$ (top) and $B$ (bottom) of the perovskite $A B X_{3}$ in the initial list of fluoride (red) and oxide (blue) paramagnetic semiconductors and after screening for mechanical stability (violet and cyan, respectively).

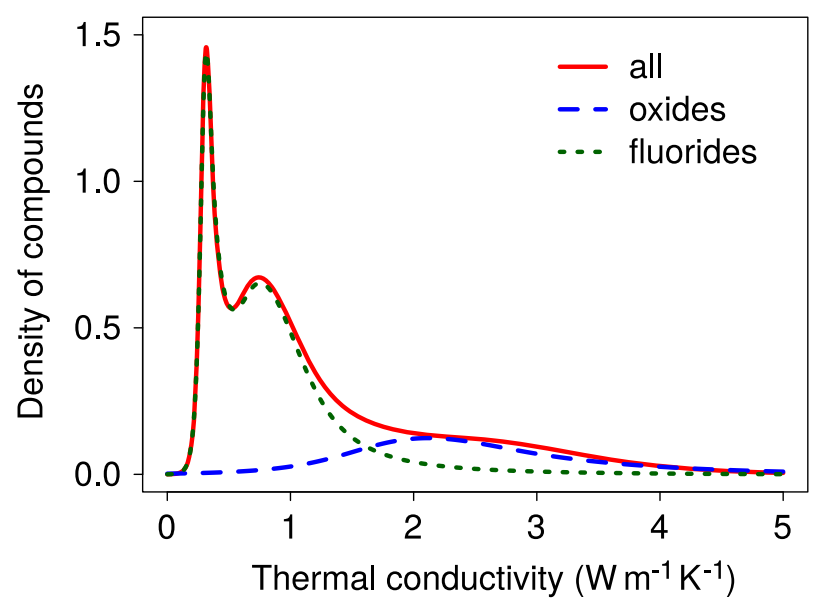

FIG. 6. Distribution of compounds as a function of the lattice thermal conductivity at $1000 \mathrm{~K}$. The red curve corresponds to the distribution for all mechanically stable compounds. The blue curve corresponds to the distribution for oxides only. The green curve corresponds to the distribution for fluorides only.

Figure 7 displays the correlograms for fluorides and oxides between the following variables: the thermal conductivity $\kappa$, the thermal conductivity in the small grain limit $\kappa_{\mathrm{sg}}[3,96]$, the mean phonon group velocity $v_{g}$, the heat capacity $c_{V}$, the root-mean-square Grüneisen parameter $\gamma_{\mathrm{rms}}$ [97,98], the masses of atoms at sites $A$ and $B$ of the perovskite $A B X_{3}$, their electronegativities, their Pettifor numbers [99], their ionic radii, the lattice parameter of the compound, and its electronic band gap. Remarkably, sites $A$ and $B$ play very different roles in fluorides and oxides. In particular, the thermal conductivity of fluorides is mostly influenced by substitutions of the atom inside the fluorine octahedron (site $B$ ), while the interstitial atom at site $A$ has a negligible impact. The opposite is true for the oxides. This means that when searching for new compounds with a low lattice thermal conductivity, substitutions at the $A$ site of fluorides can be performed to optimize cost or other considerations without impacting thermal transport.

Common to both fluorides and oxides, the lattice parameter is mostly correlated with the ionic radius of atom $B$ rather than atom $A$. Interestingly, the lattice parameter is larger for fluorides, although the ionic radius of fluorine is smaller than for oxygen. This is presumably due to partially covalent bonding in oxides (see, e.g., Ref. [100]). In contrast, fluorides are more ionic: the mean degree of ionicity of the $X-B$ bond calculated from Pauling's electronegativities [101] $e_{X}$ and $e_{B}$ as $I_{X B}=$ $100\left(1-e^{\left(e_{X}-e_{B}\right) / 4}\right)$ yields a value of $56 \%$ for oxides versus $74 \%$ for fluorides. Ionicity is also reflected by the band structure, as can be seen from the weak dispersion and hybridization of the $F-2 p$ bands [102]. This may explain why the role of atoms at sites $A$ and $B$ is so different between the two types of perovskites. We think that the more ionic character combined with the small nominal 

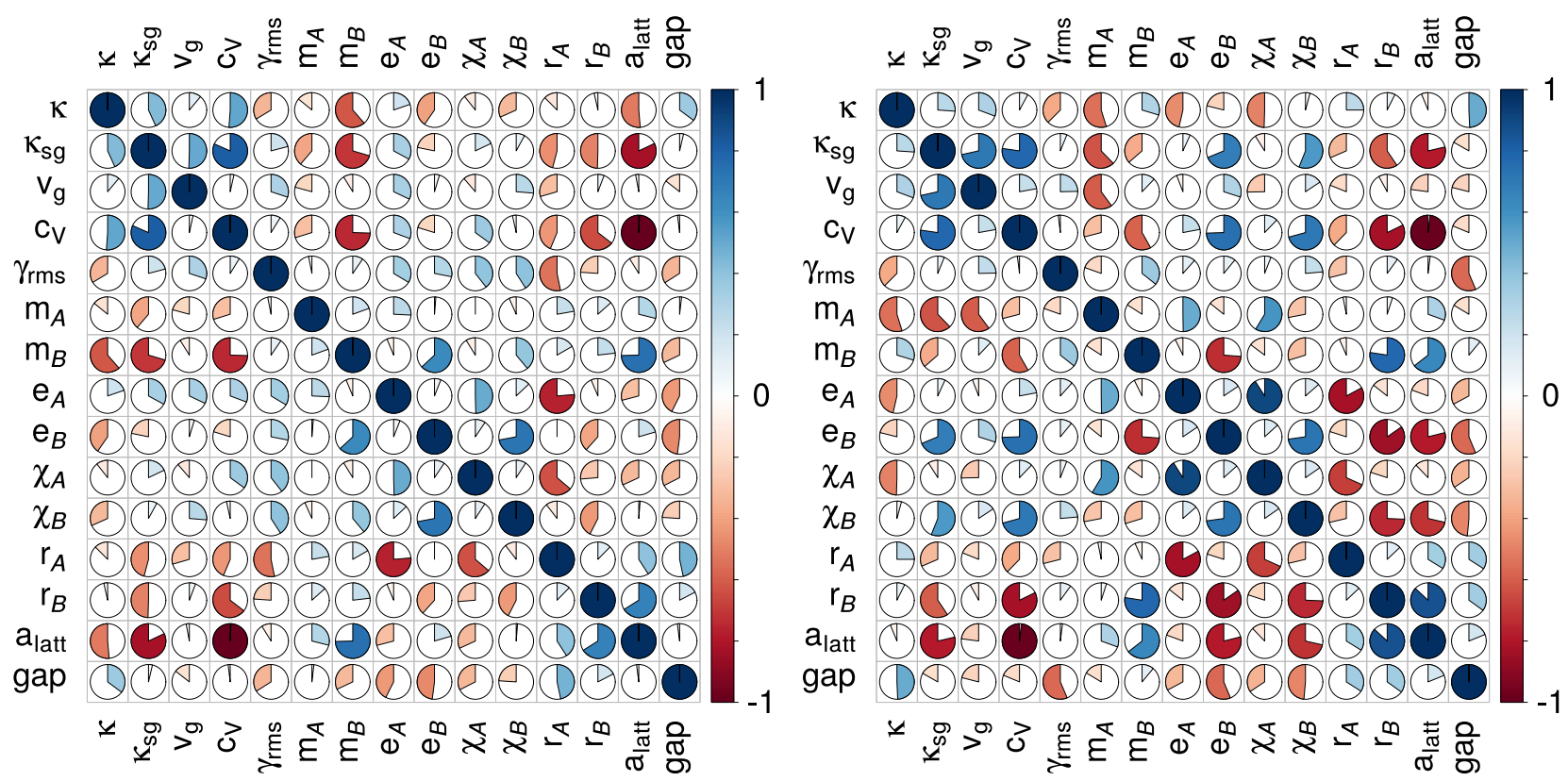

FIG. 7. Correlograms between the thermal conductivity $\kappa$, the thermal conductivity in the small grain limit $\kappa_{\mathrm{sg}}$, the mean phonon group velocity $v_{g}$, the heat capacity $c_{V}$, the root-mean-square Grüneisen parameter $\gamma_{\mathrm{rms}}$, the masses $m_{A}$ and $m_{B}$ of atoms at sites $A$ and $B$ of the perovskite $A B X_{3}$, their electronegativities $e_{A}, e_{B}$, their Pettifor scales $\chi_{A}, \chi_{B}$, their ionic radii $r_{A}, r_{B}$, the lattice parameter of the compound $a_{\text {latt }}$, and its electronic band gap, for mechanically stable fluorides (left) and oxides (right) at $1000 \mathrm{~K}$.

charge in fluorides makes the octahedron cage enclosing atom $B$ less rigid, such that the influence of atom $B$ on the thermal conductivity becomes more significant.

It is interesting to note that the electronic band gap is largely correlated with the electronegativity of atom $B$, suggesting the first electronic excitations likely involve electron transfer from the anion to atom $B$ atom. This means that the size of the band gap is essentially a measure of ionicity in these compounds, and indeed, fluorides have much larger band gaps than oxides in general. Furthermore, the band gap is also negatively correlated with the root-meansquare Grüneisen parameter. As a consequence, it is positively correlated with the lattice thermal conductivity-but only within each family, since when we compare oxides with fluorides the more important factors described above come into play. This implies that the phonon frequencies of a more ionic semiconductor are less modified by volume changes, due to its smaller polarizability. Such a phenomenon is illustrated experimentally in the family of alkali halides: when the halogen is substituted along the series from iodine to fluorine for a given alkali metal, the ionicity of the material increases. As a result, the band gap increases [105] and the high-temperature Grüneisen parameter decreases [106,107]. Also, the thermal conductivity increases [108], but disentangling the effect of ionicity from other influences-such as the lighter mass - becomes cumbersome. On the other hand, if we turn to the less ionic $\mathrm{CuCl}$ [109], we indeed find that it has a smaller thermal conductivity than the alkali chlorides [110], along with a smaller band gap of $3.4 \mathrm{eV}$. We note that such an effect in which less localized electrons reduce the thermal conductivity due to higher polarizability was also described in a comparison of zinc blende III-V with rocksalt IV-VI compounds [111].

\section{CONCLUSION}

Employing finite-temperature $a b$ initio calculations of force constants in combination with machine-learning techniques, we assess the mechanical stability and thermal conductivity of hundreds of oxides and fluorides with cubic perovskite structures at high temperatures. We show that the thermal conductivities of fluorides are generally much smaller than those of oxides, and we find new potentially stable perovskite compounds. We also show that the thermal conductivity of cubic perovskites generally decreases more slowly than the inverse of temperature. Finally, we provide simple ways of tuning the thermal properties of oxides and fluorides by contrasting the effects of substitutions at the $A$ and $B$ sites. We hope that this work will trigger further interest in halide perovskites for applications that require low thermal conductivity.

\section{ACKNOWLEDGMENTS}

This work is partially supported by the French Association Instituts Carnot (project SIEVE) and the European Union's Horizon 2020 Research and Innovation Programme [Grant No. 645776 (ALMA)]. S. C. acknowledges support from DOD-ONR (N00014-13-1-0030, N00014-13-1-0635), DOE (DE-AC02-05CH11231, specifically BES Grant No. EDCBEE), and the Duke University Center for Materials 
Genomics. C. O. acknowledges support from the National Science Foundation Graduate Research Fellowship under Grant No. DGF1106401. AFLOW calculations were performed at the Duke University Center for Materials Genomics. We also acknowledge the CRAY corporation for computational support.

[1] S. Curtarolo, G. L. Hart, M. B. Nardelli, N. Mingo, S. Sanvito, and O. Levy, The High-Throughput Highway to Computational Materials Design, Nat. Mater. 12, 191 (2013).

[2] D. G. Cahill et al., Nanoscale Thermal Transport. II. 2003-2012, Appl. Phys. Rev. 1, 011305 (2014).

[3] J. Carrete, W. Li, N. Mingo, S. Wang, and S. Curtarolo, Finding Unprecedentedly Low-Thermal-Conductivity Half-Heusler Semiconductors via High-Throughput Materials Modeling, Phys. Rev. X 4, 011019 (2014).

[4] A. Seko, A. Togo, H. Hayashi, K. Tsuda, L. Chaput, and I. Tanaka, Prediction of Low-Thermal-Conductivity Compounds with First-Principles Anharmonic LatticeDynamics Calculations and Bayesian Optimization, Phys. Rev. Lett. 115, 205901 (2015).

[5] L. D. Landau and E. M. Lifshitz, Course of Theoretical Physics (Pergamon Press, New York, 1969), Vol. 5.

[6] C. J. Howard and H. T. Stokes, Structures and Phase Transitions in Perovskites-A Group-Theoretical Approach, Acta Crystallogr. Sect. A 61, 93 (2005).

[7] H. Thomas and K. A. Müller, Structural Phase Transitions in Perovskite-Type Crystals, Phys. Rev. Lett. 21, 1256 (1968).

[8] W. Cochran and A. Zia, Structure and Dynamics of Perovskite-Type Crystals, Phys. Status Solidi B 25, 273 (1968).

[9] R. J. Angel, J. Zhao, and N. L. Ross, General Rules for Predicting Phase Transitions in Perovskites due to Octahedral Tilting, Phys. Rev. Lett. 95, 025503 (2005).

[10] S. Curtarolo, W. Setyawan, G. L. W. Hart, M. Jahnatek, R. V. Chepulskii, R. H. Taylor, S. Wang, J. Xue, K. Yang, O. Levy, M. J. Mehl, H. T. Stokes, D. O. Demchenko, and D. Morgan, AFLOW: an Automatic Framework for HighThroughput Materials Discovery, Comput. Mater. Sci. 58, 218 (2012).

[11] R. H. Taylor, F. Rose, C. Toher, O. Levy, K. Yang, M. B. Nardelli, and S. Curtarolo, A RESTful API for Exchanging Materials Data in the AFLOWLIB.org Consortium, Comput. Mater. Sci. 93, 178 (2014).

[12] See Supplemental Material at http://link.aps.org/ supplemental/10.1103/PhysRevX.6.041061 for further details about the method and the lists of compounds, a comprehensive list of phonon spectra, and statistical assessments of the performance of the machine learning methods used.

[13] C. E. Calderon, J. J. Plata, C. Toher, C. Oses, O. Levy, M. Fornari, A. Natan, M. J. Mehl, G. L. W. Hart, M. B. Nardelli, and S. Curtarolo, The AFLOW Standard for High-Throughput Materials Science Calculations, Comput. Mater. Sci. 108, 233 (2015).
[14] P. Souvatzis, O. Eriksson, M. I. Katsnelson, and S. P. Rudin, Entropy Driven Stabilization of Energetically Unstable Crystal Structures Explained from First Principles Theory, Phys. Rev. Lett. 100, 095901 (2008).

[15] O. Hellman, I. A. Abrikosov, and S. I. Simak, Lattice Dynamics of Anharmonic Solids from First Principles, Phys. Rev. B 84, 180301 (2011).

[16] O. Hellman and I. A. Abrikosov, Temperature-Dependent Effective Third-Order Interatomic Force Constants from First Principles, Phys. Rev. B 88, 144301 (2013).

[17] I. Errea, M. Calandra, and F. Mauri, First-Principles Theory of Anharmonicity and the Inverse Isotope Effect in Superconducting Palladium-Hydride Compounds, Phys. Rev. Lett. 111, 177002 (2013).

[18] T. Tadano and S. Tsuneyuki, Self-Consistent Phonon Calculations of Lattice Dynamical Properties in Cubic $\mathrm{SrTiO}_{3}$ with First-Principles Anharmonic Force Constants, Phys. Rev. B 92, 054301 (2015).

[19] A. van Roekeghem, J. Carrete, and N. Mingo, Anomalous Thermal Conductivity and Suppression of Negative Thermal Expansion in $\mathrm{ScF}_{3}$, Phys. Rev. B 94, 020303(R) (2016).

[20] W. Li, J. Carrete, N. A. Katcho, and N. Mingo, ShengBTE: A Solver of the Boltzmann Transport Equation for Phonons, Comput. Phys. Commun. 185, 1747 (2014).

[21] T. Komabayashi, K. Hirose, N. Sata, Y. Ohishi, and L. S. Dubrovinsky, Phase Transition in $\mathrm{CaSiO}_{3}$ Perovskite, Earth Planet. Sci. Lett. 260, 564 (2007).

[22] A. I. Lebedev, Ferroelectric Properties of $\mathrm{RbNbO}_{3}$ and $\mathrm{RbTaO}_{3}$, Phys. Solid State 57, 331 (2015).

[23] W. L. W. Ludekens and A. J. E. Welch, Reactions between Metal Oxides and Fluorides: Some New Double-Fluoride Structures of Type $A B \mathrm{~F}_{3}$, Acta Crystallogr. 5, 841 (1952).

[24] C. Ridou, M. Rousseau, J. Y. Gesland, J. Nouet, and A. Zarembowitch, The $193 \mathrm{~K}$ Phase Transition in $\mathrm{RbCaF}_{3}$, Ferroelectrics 12, 199 (1976).

[25] J. Martin, in Phonon Scattering in Solids, edited by L. Challis, V. Rampton, and A. Wyatt (Springer, New York, 1976), pp. 258-260.

[26] B. J. Kennedy, A. K. Prodjosantoso, and C. J. Howard, Powder Neutron Diffraction Study of the High Temperature Phase Transitions in $\mathrm{NaTaO}_{3}$, J. Phys. Condens. Matter 11, 6319 (1999).

[27] M. Rousseau, J. Y. Gesland, J. Julliard, J. Nouet, J. Zarembowitch, and A. Zarembowitch, Crystallographic, Elastic, and Raman Scattering Investigations of Structural Phase Transitions in $\mathrm{RbCdF}_{3}$ and $\mathrm{TlCdF}_{3}$, Phys. Rev. B 12, 1579 (1975).

[28] A. Zanardi, M. A. Novikov, E. Martin, J. Benet-Buchholz, and V. V. Grushin, Direct Cupration of Fluoroform, J. Am. Chem. Soc. 133, 20901 (2011).

[29] W. Xiao, D. Tan, W. Zhou, J. Liu, and J. Xu, Cubic Perovskite Polymorph of Strontium Metasilicate at High Pressures, Am. Mineral. 98, 2096 (2013).

[30] J. Kwapulinski, M. Pawełczyk, and J. Dec, On the Pb Thermal Vibrations in $\mathrm{PbHfO}_{3}$ Crystals, J. Phys. Condens. Matter 6, 4655 (1994).

[31] G. Shirane, R. Newnham, and R. Pepinsky, Dielectric Properties and Phase Transitions of $\mathrm{NaNbO}_{3}$ and (Na, K) $\mathrm{NbO}_{3}$, Phys. Rev. 96, 581 (1954). 
[32] S. K. Mishra, R. Mittal, V. Y. Pomjakushin, and S. L. Chaplot, Phase Stability and Structural Temperature Dependence in Sodium Niobate: A High-Resolution Powder Neutron Diffraction Study, Phys. Rev. B 83, 134105 (2011).

[33] M. Tachibana, T. Kolodiazhnyi, and E. TakayamaMuromachi, Thermal Conductivity of Perovskite Ferroelectrics, Appl. Phys. Lett. 93, 092902 (2008).

[34] J. Portier, A. Tressaud, and J.-L. Dupin, Les Pérovskites Fluorées $\mathrm{AgMeF}_{3}(\mathrm{Me}=\mathrm{Mg}, \mathrm{Mn}, \mathrm{Co}, \mathrm{Ni}, \mathrm{Cu}, \mathrm{Zn}), \mathrm{Cr}$. Acad. Sci. C 270, 216 (1970).

[35] T. Maekawa, K. Kurosaki, and S. Yamanaka, Thermal and Mechanical Properties of Perovskite-Type Barium Hafnate, J. Alloys Compd. 407, 44 (2006).

[36] W. Pies and A. Weiss, in Key Elements: F, Cl, Br, I (Springer-Verlag, Berlin, 1973), pp. 104-115.

[37] M. Kestigian, F. D. Leipziger, W. J. Croft, and R. Guidoboni, Preparation, Single Crystal Growth, and Crystallographic Properties of $\mathrm{FeF}_{2}, \mathrm{RbFeF}_{3}$, and $\mathrm{CsFeF}_{3}$, Inorg. Chem. 5, 1462 (1966).

[38] N. Ramadass, T. Palanisamy, J. Gopalakrishnan, G. Aravamudan, and M. Sastri, Some $\mathrm{ABO}_{3}$ Oxides with Defect Pyrochlore Structure, Solid State Commun. 17, 545 (1975).

[39] M. Arakawa, A. Okamoto, H. Ebisu, and H. Takeuchi, An Electron Paramagnetic Resonance Study of $\mathrm{Fe}^{3+}$ Centres in $\mathrm{Tl}_{2} \mathrm{MgF}_{4}$ and $\mathrm{Tl}_{2} \mathrm{ZnF}_{4}$ Crystals, J. Phys. Condens. Matter 18, 3053 (2006).

[40] A. Kania and A. Ratuszna, Phase Transitions in $\mathrm{AgTaO}_{3}$ Single Crystals, Phase Transit. 2, 7 (1981).

[41] M. Pawełczyk, Phase Transitions in $\mathrm{AgTa}_{x} \mathrm{Nb}_{1-x} \mathrm{O}_{3}$ Solid Solutions, Phase Transit. 8, 273 (1987).

[42] D. Demetriou, C. Catlow, A. Chadwick, G. Mclntyre, and I. Abrahams, The Anion Disorder in the Perovskite Fluoride $\mathrm{KCaF}_{3}$, Solid State Ionics 176, 1571 (2005).

[43] I. G. Wood, K. S. Knight, G. D. Price, and J. A. Stuart, Thermal Expansion and Atomic Displacement Parameters of Cubic $\mathrm{KMgF}_{3}$ Perovskite Determined by HighResolution Neutron Powder Diffraction, J. Appl. Crystallogr. 35, 291 (2002).

[44] J. Xu, Ph.D. thesis, Georgia Institute of Technology, 2000.

[45] R. Armiento, B. Kozinsky, M. Fornari, and G. Ceder, Screening for High-Performance Piezoelectrics Using High-Throughput Density Functional Theory, Phys. Rev. B 84, 014103 (2011).

[46] I. E. Castelli, T. Olsen, S. Datta, D. D. Landis, S. Dahl, K. S. Thygesen, and K.W. Jacobsen, Computational Screening of Perovskite Metal Oxides for Optimal Solar Light Capture, Energy Environ. Sci. 5, 5814 (2012).

[47] M. Rousseau, J. Gesland, B. Hennion, G. Heger, and B. Renker, Low Energy Phonon Dispersion Curves of $\mathrm{KZnF}_{3}$ and $\mathrm{CsCaF}_{3}$, Solid State Commun. 38, 45 (1981).

[48] R. Hoppe and R. Homann, Über $\mathrm{CsHgF}_{3}, \mathrm{RbHgF}_{3}$ und $\mathrm{KHgF}_{3}$, Z. Anorg. Allg. Chem. 369, 212 (1969).

[49] B. A. Strukov, S. T. Davitadze, S. N. Kravchun, S. A. Taraskin, M. Goltzman, V. V. Lemanov, and S. G. Shulman, Specific Heat and Heat Conductivity of $\mathrm{BaTiO}_{3}$ Polycrystalline Films in the Thickness Range 20-1100 nm, J. Phys. Condens. Matter 15, 4331 (2003).
[50] Y. Uetsuji, S. Kumazawa, T. Ohnishi, K. Tsuchiya, and E. Nakamachi, Structure Evaluation of Bio-Compatible Lead-Free Piezoelectric Materials by Crystal System Distinction and First Principles Calculations, Trans. Jpn. Soc. Mech. Eng. A72, 1472 (2006).

[51] H. Muta, K. Kurosaki, and S. Yamanaka, Thermoelectric Properties of Reduced and La-Doped Single-Crystalline $\mathrm{SrTiO}_{3}$, J. Alloys Compd. 392, 306 (2005).

[52] S. R. Popuri, A. J. M. Scott, R. A. Downie, M. A. Hall, E. Suard, R. Decourt, M. Pollet, and J.-W. G. Bos, Glass-like Thermal Conductivity in $\mathrm{SrTiO}_{3}$ Thermoelectrics Induced by A-Site Vacancies, RSC Adv. 4, 33720 (2014).

[53] S. Yamanaka, T. Maekawa, H. Muta, T. Matsuda, S.-I. Kobayashi, and K. Kurosaki, Thermophysical Properties of $\mathrm{SrHfO}_{3}$ and $\mathrm{SrRuO}_{3}$, J. Solid State Chem. 177, 3484 (2004).

[54] P. Daniel, J. Toulouse, J. Y. Gesland, and M. Rousseau, Raman-Scattering Investigation of the Hexagonal Perovskite $\mathrm{RbZnF}_{3}$, Phys. Rev. B 52, 9129 (1995).

[55] B. J. Kennedy, C. J. Howard, and B. C. Chakoumakos, High-Temperature Phase Transitions in $\mathrm{SrHfO}_{3}$, Phys. Rev. B 60, 2972 (1999).

[56] T. Thao Tran and P. Shiv Halasyamani, Synthesis and Characterization of $A \mathrm{SnF}_{3}\left(A=\mathrm{Na}^{+}, \mathrm{K}^{+}, \mathrm{Rb}^{+}, \mathrm{Cs}^{+}\right)$, J. Solid State Chem. 210, 213 (2014).

[57] S. Yamanaka, T. Hamaguchi, T. Oyama, T. Matsuda, S.-i. Kobayashi, and K. Kurosaki, Heat Capacities and Thermal Conductivities of Perovskite Type $\mathrm{BaZrO}_{3}$ and $\mathrm{BaCeO}_{3}$, J. Alloys Compd. 359, 1 (2003).

[58] H. Yusa, N. Sata, and Y. Ohishi, Rhombohedral (9R) and Hexagonal (6H) Perovskites in Barium Silicates under High Pressure, Am. Mineral. 92, 648 (2007).

[59] M. Łukaszewski, M. Pawełczyk, J. Haňderek, and A. Kania, On the Phase Transitions in Silver Niobate $\mathrm{AgNbO}_{3}$, Phase Transit. 3, 247 (1983).

[60] P. Sciau, A. Kania, B. Dkhil, E. Suard, and A. Ratuszna, Structural Investigation of $\mathrm{AgNbO}_{3}$ Phases Using X-Ray and Neutron Diffraction, J. Phys. Condens. Matter 16, 2795 (2004).

[61] Y. Yamane, K. Yamada, and K. Inoue, Mechanochemical Synthesis and Order-Disorder Phase Transition in Fluoride Ion Conductor $\mathrm{RbPbF}_{3}$, Solid State Ionics $\mathbf{1 7 9}$, 605 (2008).

[62] A. Okazaki and Y. Suemune, The Crystal Structures of $\mathrm{KMnF}_{3}, \mathrm{KFeF}_{3}, \mathrm{KCoF}_{3}, \mathrm{KNiF}_{3}$ and $\mathrm{KCuF}_{3}$ Above and Below Their Néel Temperatures, J. Phys. Soc. Jpn. 16, 671 (1961).

[63] Y. Suemune, The Anomalous Thermal Conduction in $\mathrm{KFeF}_{3}$ Single Crystal at Low Temperatures, J. Phys. Soc. Jpn. 19, 2234 (1964).

[64] B. L. Clark and D. A. Keszler, Hydrothermal Dehydration of Precipitates: Convenient Synthesis Method for Solids, Inorg. Chem. 40, 1724 (2001).

[65] R. Armiento, B. Kozinsky, G. Hautier, M. Fornari, and G. Ceder, High-Throughput Screening of Perovskite Alloys for Piezoelectric Performance and Thermodynamic Stability, Phys. Rev. B 89, 134103 (2014).

[66] M. Hidaka, S. Hosogi, M. Ono, and K. Horai, Structural Phase Transitions in $\mathrm{KCdF}_{3}$, Solid State Commun. 23, 503 (1977). 
[67] M. Hidaka, Z. Zhou, and S. Yamashita, Structural Phase Transitions in $\mathrm{KCdF}_{3}$ and $\mathrm{K}_{0.5} \mathrm{Rb}_{0.5} \mathrm{CdF}_{3}$, Phase Transit. 20, 83 (1990).

[68] S. Hull and P. Berastegui, Superionic Phases in the $\left(\mathrm{PbF}_{2}\right)_{1-x}-(M \mathrm{~F})_{x}, M=\mathrm{K}, \mathrm{Rb}$ and $\mathrm{Cs}$, systems, J. Phys. Condens. Matter 11, 5257 (1999).

[69] A. Mackay, The Unit Cell and Space-Group of Alamosite $\left(\mathrm{PbSiO}_{3}\right)$, Mineral. Mag. 29, 933 (1952).

[70] W. Xiao, D. Tan, W. Zhou, M. Chen, X. Xiong, M. Song, J. Liu, H.-K. Mao, and J. Xu, A New Cubic Perovskite in $\mathrm{PbGeO}_{3}$ at High Pressures, Am. Mineral. 97, 1193 (2012).

[71] M. Duarte, M. Vieira, and S. Baldochi, Thermal Diffusivity of $\mathrm{BaLiF}_{3}$ Crystals, Mater. Sci. Eng. B 25, 133 (1994).

[72] M. Mortier, J. Gesland, and M. Rousseau, Experimental and Theoretical Study of Second-Order Raman Scattering in $\mathrm{BaLiF}_{3}$, Solid State Commun. 89, 369 (1994).

[73] X.-N. Wu, X.-N. Li, X.-L. Ding, and S.-G. He, Activation of Multiple $\mathrm{C}-\mathrm{H}$ Bonds Promoted by Gold in $\mathrm{AuNbO}_{3}^{+}$ Clusters, Angew. Chem., Int. Ed. Engl. 52, 2444 (2013).

[74] M. Wildner and G. Giester, Crystal Structures of $\mathrm{SrSeO}_{3}$ and $\mathrm{CaSeO}_{3}$ and Their Respective Relationships with Molybdomenite- and Monazite-Type Compounds-An Example for Stereochemical Equivalence of $\mathrm{ESeO}_{3}$ Groups ( $E=$ Lone Electron Pair) with Tetrahedral $\mathrm{TO}_{4}$ Groups, Neues Jb. Miner. Abh. 184, 29 (2007).

[75] H. O'Daniel and L. Tscheischwili, Zur Struktur von $\mathrm{NaBeF}_{3}$ und $\beta-\mathrm{CaSiO}_{3}$, Neues Jb. Miner. Abh. 1945-1948, 56 (1945).

[76] D. M. Roy, R. Roy, and E. F. Osborn, Fluoride Model Systems: III, The System $\mathrm{NaF}-\mathrm{BeF}_{2}$ and the Polymorphism of $\mathrm{Na}_{2} \mathrm{BeF}_{4}$ and $\mathrm{BeF}_{2}$, J. Am. Ceram. Soc. 36, 185 (1953).

[77] J. Foulon, J. Durand, A. Larbot, L. Cot, and A. Soufiane, Crystal Structures of $\mathrm{MSnF}_{3}$ for $M: \mathrm{K}, \mathrm{Rb}, \mathrm{Tl}$; Ionic Mobility, Eur. J. Solid State Inorg. Chem. 30, 87 (1993).

[78] M. Shafer and T. McGuire, Preparation and Properties of Ferrimagnets in the $\mathrm{RbMgF}_{3}-\mathrm{RbCoF}_{3}$ System, J. Phys. Chem. Solids 30, 1989 (1969).

[79] C. Hebecker, Neue Ternäre Fluoride mit Einwertigem Thallium und Silber als Kationen, Naturwiss. 60, 154 (1973).

[80] Y. Suemune and H. Ikawa, Thermal Conductivity of $\mathrm{KMnF}_{3}, \mathrm{KCoF}_{3}, \mathrm{KNiF}_{3}$, and $\mathrm{KZnF}_{3}$ Single Crystals, J. Phys. Soc. Jpn. 19, 1686 (1964).

[81] J. Longo and J. Kafalas, The Effect of Pressure and B-Cation Size on the Crystal Structure of $\mathrm{CsBF}_{3}$ Compounds $(B=\mathrm{Mn}, \mathrm{Fe}, \mathrm{Co}, \mathrm{Ni}, \mathrm{Zn}, \mathrm{Mg})$, J. Solid State Chem. 1, 103 (1969).

[82] I. I. Buchinskaya and P. P. Fedorov, Lead Difluoride and Related Systems, Russ. Chem. Rev. 73, 371 (2004).

[83] D. Babel, Structure and Bonding, (Springer, Berlin, 1967), pp. $1-87$.

[84] E. Grüneisen, Theorie des Festen Zustandes Einatomiger Elemente, Ann. Phys. (Berlin) 344, 257 (1912).

[85] N. Ashcroft and N. Mermin, Solid State Physics (Saunders College, Philadelphia, 1976).
[86] J. Fabian and P. B. Allen, Thermal Expansion and Grüneisen Parameters of Amorphous Silicon: A Realistic Model Calculation, Phys. Rev. Lett. 79, 1885 (1997).

[87] D. A. Broido, A. Ward, and N. Mingo, Lattice Thermal Conductivity of Silicon from Empirical Interatomic Potentials, Phys. Rev. B 72, 014308 (2005).

[88] B. K. Greve, K. L. Martin, P. L. Lee, P. J. Chupas, K. W. Chapman, and A.P. Wilkinson, Pronounced Negative Thermal Expansion from a Simple Structure: Cubic $\mathrm{ScF}_{3}$, J. Am. Chem. Soc. 132, 15496 (2010).

[89] R. Peierls, Zur Kinetischen Theorie der Wärmeleitung in Kristallen, Ann. Phys. (Berlin) 395, 1055 (1929).

[90] M. C. Roufosse and P. G. Klemens, Lattice Thermal Conductivity of Minerals at High Temperatures, J. Geophys. Res. 79, 703 (1974).

[91] C. J. Glassbrenner and G. A. Slack, Thermal Conductivity of Silicon and Germanium from $3{ }^{\circ} \mathrm{K}$ to the Melting Point, Phys. Rev. 134, A1058 (1964).

[92] T. Maekawa, K. Kurosaki, and S. Yamanaka, Thermal and Mechanical Properties of Polycrystalline $\mathrm{BaSnO}_{3}$, J. Alloys Compd. 416, 214 (2006).

[93] However, we note that transitions to other structures can take place, in particular, to one of hexagonal symmetry, such as in $\mathrm{BaTiO}_{3}$ [94], $\mathrm{RbZnF}_{3}$ [54] or $\mathrm{RbMgF}_{3}$ [78]. This phase transition is of first order, in contrast to displacive transitions that are of second order.

[94] R. M. Glaister and H. F. Kay, An Investigation of the Cubic-Hexagonal Transition in Barium Titanate, Proc. Phys. Soc. 76, 763 (1960).

[95] Precision is defined as the fraction of true positives in all positives reported by the model and recall is defined as the fraction of true positives found using the model with respect to all true positives.

[96] J. Carrete, N. Mingo, S. Wang, and S. Curtarolo, Nanograined Half-Heusler Semiconductors as Advanced Thermoelectrics: An Ab Initio High-Throughput Statistical Study, Adv. Funct. Mater. 24, 7427 (2014).

[97] L. Bjerg, B. B. Iversen, and G. K. H. Madsen, Modeling the Thermal Conductivities of the Zinc Antimonides $\mathrm{ZnSb}$ and $\mathrm{Zn}_{4} \mathrm{Sb}_{3}$, Phys. Rev. B 89, 024304 (2014).

[98] G. K. H. Madsen, A. Katre, and C. Bera, Calculating the Thermal Conductivity of the Silicon Clathrates Using the Quasi-Harmonic Approximation, Phys. Status Solidi A 213, 802 (2016).

[99] D. Pettifor, A Chemical Scale for Crystal-Structure Maps, Solid State Commun. 51, 31 (1984).

[100] A. Koleżyński and K. Tkacz-Śmiech, From the Molecular Picture to the Band Structure of Cubic and Tetragonal Barium Titanate, Ferroelectrics 314, 123 (2005).

[101] L. Pauling, The Nature of the Chemical Bond. IV. The Energy of Single Bonds and the Relative Electronegativity of Atoms, J. Am. Chem. Soc. 54, 3570 (1932).

[102] See, for instance, the band structure of $\mathrm{SrTiO}_{3}$ [103] compared to the one of $\mathrm{KCaF}_{3}$ [104]. In those two compounds, the degree of ionicity of the $X$ - $B$ bond calculated from Pauling's electronegativity is $59 \%$ and $89 \%$, respectively. 
[103] K. van Benthem, C. Elsässer, and R. H. French, Bulk Electronic Structure of $\mathrm{SrTiO}_{3}$ : Experiment and Theory, J. Appl. Phys. 90, 6156 (2001).

[104] B. Ghebouli, M. Fatmi, M. Ghebouli, H. Choutri, L. Louail, T. Chihi, A. Bouhemadou, and S. Bin-Omran, Theoretical Study of the Structural, Elastic, Electronic and Optical properties of $\mathrm{XCaF}_{3}(X=\mathrm{K}$ and $\mathrm{Rb})$, Solid State Sci. 43, 9 (2015).

[105] R. T. Poole, J. G. Jenkin, J. Liesegang, and R. C. G. Leckey, Electronic Band Structure of the Alkali Halides. I. Experimental Parameters, Phys. Rev. B 11, 5179 (1975).

[106] B. N. N. Achar and G. R. Barsch, Shell Model Calculation of Thermal Expansion of Alkali Halides and Magnesium Oxide, Phys. Status Solidi A 6, 247 (1971).
[107] This is also consistent with the generally higher rootmean-square Grüneisen parameters that are found for oxides as compared to fluorides (see Supplemental Material [12]).

[108] S. Pettersson, Calculation of the Thermal Conductivity of Alkali Halide Crystals, J. Phys. C 20, 1047 (1987).

[109] A. Zunger and M. L. Cohen, Electronic Structure of $\mathrm{CuCl}$, Phys. Rev. B 20, 1189 (1979).

[110] G. A. Slack and P. Andersson, Pressure and Temperature Effects on the Thermal Conductivity of $\mathrm{CuCl}$, Phys. Rev. B 26, 1873 (1982).

[111] S. Lee, K. Esfarjani, T. Luo, J. Zhou, Z. Tian, and G. Chen, Resonant Bonding Leads to Low Lattice Thermal Conductivity, Nat. Commun. 5, 3525 (2014). 